\title{
Subjective mental time: the functional architecture of projecting the self to past and future
}

\author{
Shahar Arzy, ${ }^{1,2,3}$ Sven Collette, ${ }^{1}$ Silvio Ionta, ${ }^{1}$ Eleonora Fornari ${ }^{4}$ and Olaf Blanke ${ }^{1,3}$ \\ ${ }^{1}$ Laboratory of Cognitive Neuroscience, Brain Mind Institute, Ecole Polytechnique Fédérale de Lausanne, Station 15, \\ Lausanne 1015, Switzerland \\ ${ }^{2}$ Department of Neurology, Hadassah Hebrew University Hospital, POB 12000, Jerusalem 91120, Israel \\ ${ }^{3}$ Department of Neurology, University Hospital, Geneva 1211, Switzerland \\ ${ }^{4}$ Department of Radiology, University Hospital, Lausanne 1011, Switzerland
}

Keywords: episodic thinking, face, functional magnetic resonance imaging, human, mental time, self

\begin{abstract}
Human experience takes place in the line of mental time (MT) created through 'self-projection' of oneself to different time-points in the past or future. Here we manipulated self-projection in MT not only with respect to one's life events but also with respect to one's faces from different past and future time-points. Behavioural and event-related functional magnetic resonance imaging activity showed three independent effects characterized by (i) similarity between past recollection and future imagination, (ii) facilitation of judgements related to the future as compared with the past, and (iii) facilitation of judgements related to time-points distant from the present. These effects were found with respect to faces and events, and also suggest that brain mechanisms of MT are independent of whether actual life episodes have to be re-experienced or pre-experienced, recruiting a common cerebral network including the anteromedial temporal, posterior parietal, inferior frontal, temporo-parietal and insular cortices. These behavioural and neural data suggest that self-projection in time is a fundamental aspect of MT, relying on neural structures encoding memory, mental imagery and self.
\end{abstract}

\section{Introduction}

An essential quality of the human mind is the recall of past events and imagination of future occurrences, creating jointly the subjective 'mental time' (MT) in which everybody is living (Tulving, 1985, 2002; Atance \& O’Neill, 2001; Levine, 2004; Levine et al., 2004; Schacter et al., 2007; Suddendorf \& Corballis, 2007). It is widely accepted that past MT relies on episodic autobiographical memory, allowing the re-experience of one's personal past through the subjective 'projection' of the self to a specific time and place (Tulving, 1985, 2002; Levine, 2004; Levine et al., 2004; Buckner \& Carroll, 2007). Comparatively, future MT is thought to be based on 'episodic future thinking' or the ability to project the self forward to pre-experience an event (Atance \& O'Neill, 2001; Fellows \& Farah, 2005; Buckner \& Carroll, 2007; Gilbert \& Wilson, 2007; Suddendorf \& Corballis, 2007). This 'self-projection' involves a change of one's habitual self-location in time to the past or future; while imagining oneself in MT, one is 'projecting' oneself to different 'temporal locations' in the past and future [absolute MT (aMT)] and from these new self-locations in time one may imagine different episodes (Buckner \& Carroll, 2007; Arzy et al., 2008; Spreng et al., 2009). Moreover, from these new imagined self-locations in time, the relations of life events to the past and future are regarded differently;

Correspondence: Dr S. Arzy, ${ }^{2}$ Department of Neurology, Hadassah Hebrew University Hospital, as above.

E-mail: shahar.arzy@ekmd.huji.ac.il

Received 15 June 2009, revised 4 August 2009, accepted 29 August 2009 when imagining oneself as 8 years younger, last year's events are in the future (relative-future) in relation to the initially imagined selflocation in time and vice versa (relative-past) [relative MT (rMT)] (Arzy et al., 2008). The importance of self-projection for MT processing is also suggested by the activation of brain regions outside the classical memory areas that were found to be recruited for MT, including the posterior parietal cortex, which is probably related to visual imagery and autobiographical memory (Addis et al., 2007; Buckner \& Carroll, 2007; Szpunar et al., 2007; Arzy et al., 2008; Spreng et al., 2009), the temporo-parietal junction, which is a key region for self-related processing and self-projection (Ruby \& Decety, 2001; Blanke et al., 2005; Arzy et al., 2006, 2008; Buckner \& Carroll, 2007), and frontal regions involved in semantic and episodic memories as well as in self-processing (Gusnard et al., 2001; Addis et al., 2007; Buckner \& Carroll, 2007; Arzy et al., 2008; Spreng et al., 2009). Here we investigated the role of self-projection in time to the past and future using event-related functional magnetic resonance imaging (fMRI) with respect to two different kinds of stimuli (faces and events). In Experiment 1, participants carried out self-projection in time with respect to their own face and a familiar face (actor George Clooney) that were manipulated to depict each participant's and Clooney's face at a different age in the past and future. In Experiment 2, self-projection in time was carried out with respect to two-word descriptions of life events as employed previously by MT studies (Szpunar et al., 2007; Arzy et al., 2008; Spreng et al., 2009). Participants were asked to make judgements about past and future faces (Experiment 1) as well as events (Experiment 2) from three 


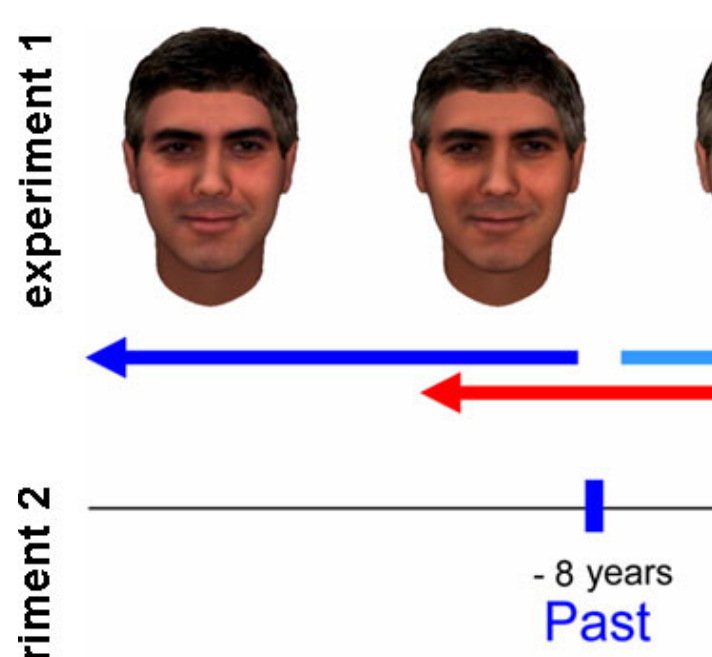

car license Challenger explosion
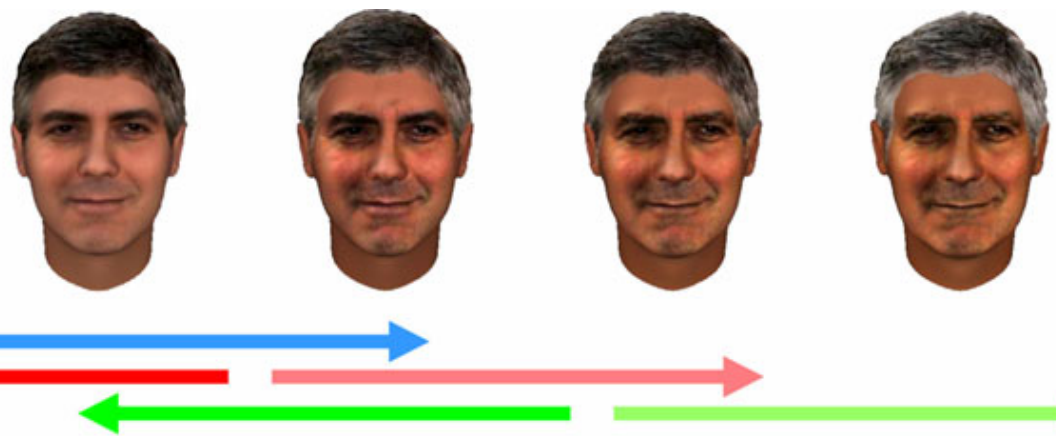
,

Actual time-point Now

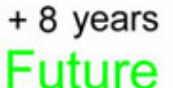

30th birthday Hurricane Katrina first child

HIV vaccination daily medications

nuclear car

FIG. 1. Stimuli and procedure. The three different self-locations in time (Past, Now and Future) are shown. Participants were asked to mentally 'project' themselves to one of these time-points and from these self-locations in time to judge if different face images (Experiment 1 , upper row) or events (Experiment 2, lower row) have already happened (relative-past, darker shades) or are yet to happen (relative-future, brighter shades). In a control task participants were asked to judge if the presented face and events are self-related (self face images and personal events, respectively) or non-self-related (Clooney's face images and world events, respectively).

different time-points: the present (Now), 8 years earlier (Past) or 8 years later (Future) (Fig. 1). This allowed us to test pre-experiencing and re-experiencing independent of the present, past or future with respect to different stimuli (faces and events) (Arzy et al., 2008). In a further control task participants were asked to make self/other discrimination judgements with respect to the same face and word stimuli without changing their self-location in MT. Behavioural measures and event-related fMRI activity showed similar patterns for faces and events recruiting almost identical neural mechanisms. These effects suggest that self-projection in time, independent of whether faces or life events are employed, is fundamental for processing of MT relying on brain mechanisms of memory, mental imagery and self.

\section{Materials and methods}

\section{Participants}

Twelve healthy volunteers (seven males, aged 29-38 years, mean \pm SD $33.7 \pm 4.2$ years) participated in the study. All participants were right handed and had normal or corrected-to-normal vision and no history of neurological or psychiatric disorders. All participants gave written informed consent before inclusion in the study, which was in accordance with the Declaration of Helsinki, and approved by the Ethical Committee of the University of Lausanne (Switzerland).

\section{Stimuli and procedures}

In Experiment 1, face images of the participant as well as a familiar person (actor George Clooney) were presented on a computer screen. Using the FACEGEN ${ }^{\circledR}$ software (Singular Inversions, Canada), these faces were modified to represent the participant's and Clooney's face appearing as 4, 12 and 20 years younger (past) or older (future) (Fig. 1, upper row). In Experiment 2, two-word phrases describing different common events from personal life (e.g. car licence, first child) or non-personal world events (e.g. Challenger explosion, hurricane Katrina) known to the participants were presented (Fig. 1, lower row). In the experimental manipulation we asked participants to imagine themselves in each session at one of three different selflocations in time: Now (the present time), Past ( 8 years in the past) or Future ( 8 years in the future). The different self-locations in time were tested in separate sessions. In Experiment 1, faces of the participant or of Clooney were shown and participants were asked to indicate whether the presented face represented the participant's/Clooney's appearance before (relative-past) or after (relative-future) the imagined self-location in time. For example, in the Now or Future conditions, the participant should indicate 'relative-past' for their face image depicting their appearance 4 years ago but in the Past condition, where their imagined self-location in time is 8 years ago, they should indicate 'relative-future' for the same face. In Experiment 2, participants were asked to indicate whether the presented event from their personal life or world history occurred before or after the currently imagined self-location in time. Likewise, for example, in Experiment 2, in the Now or Future conditions the participant should indicate 'relative-past' for the hurricane Katrina but in the Past condition they should indicate 'relative-future' because it has not been 8 years since the event. However, if they are anticipating their first child in the next few years they should indicate 'relative-future' for 'first child' in the Past and Now conditions but 'relative-past' in the Future condition. Thus, we examined MT dependently and independently from the imagined self-location in time (Past, Now or Future). As the same face/event could be relatively in the past or in the future with respect to the imagined temporal self-location (Arzy et al., 2008), we labelled this effect 'rMT', independent of the imagined selflocation in time. Self-projection to different points in time was labelled aMT, dependent on the imagined self-location in time. Finally, in a control discrimination task, participants were asked to indicate if the same faces/events were self-related (self face in Experiment 1; personal events in Experiment 2) or non-self-related (Clooney's face in Experiment 1; world events in Experiment 2) without changing their habitual self-location in time.

Stimuli $\left[\mathrm{FACEGEN}^{\circledR}\right.$-generated faces and two-word phrases with average length of $13.6 \pm 2.5$ letters (mean $\pm \mathrm{SD}$ )] were designed to be 
in the range of \pm 20 years of the imagined self-location in time. Stimuli appeared for $700 \mathrm{~ms}$ in the centre of the computer screen (presented on $800 \times 600$ pixels Nordic NeuroLab Visual System ${ }^{\circledR}$ OLED goggles) with a jittered inter-stimulus interval ranging between 2300 and $5300 \mathrm{~ms}$. Judgements were given using the index and middle fingers of the left and right hand in alternating blocks and counterbalanced across subjects as a button press on a serial response box. Participants were instructed to respond as quickly and precisely as possible. The two experiments were performed in four sessions each (past, now, future and control) and counterbalanced across subjects. Each session included 64 stimuli, equally distributed among four groups: self (self faces/personal events) in relative-past, self in relative-future, non-self (Clooney's faces/world events) in relativepast and non-self in relative-future, appearing in random order.

\section{Analysis of behavioural data}

Subsequent to the experiment, participants were asked to approximate the dates of the presented events. Correct responses for Experiment 2 were determined according to the estimated dates, as provided separately by each participant. Repeated-measures ANOVAs were run between participants on reaction time (RT) and error rate (ER) with the factors aMT (past, now and future), rMT (relative-past and relativefuture) and Self (self-related and non-self-related). Two-tailed paired $t$-tests were run between participants on RTs and ERs for the control tasks between self-related and non-self-related faces (Experiment 1) and events (Experiment 2).

\section{Recording and analysis of functional magnetic resonance imaging data}

In the present experiment event-related fMRI images were collected with a Siemens Trio 3T system with EPI gradient echo T2-weighted sequence on a 12 channel head coil at The Centre Hospitalier Universitaire Vaudois and the Center for Biomedical Imaging of Geneva and Lausanne, Switzerland. Each experimental session included the acquisition of 108 volumes of 28 consecutive slices each and covering the whole brain (TR, $3 \mathrm{~s}$; TE, $60 \mathrm{~ms}$; $64 \times 64$ image matrix; $3.5 \times 3.5 \times 3.5 \mathrm{~mm}$ isotropic voxels). The acquisition plane was oriented parallel to the anterior/posterior commissure. As a structural basis for activation display, a sagittal T1-weighted threedimensional gradient echo sequence (MPRAGE) was acquired using a Siemens multiplanar rapid acquisition gradient echo sequence $(1 \mathrm{~mm}$ isotropic voxels; 160 sagittal slices; TR, $9.7 \mathrm{~ms}$; TE, $4 \mathrm{~ms})$. The subject's head was cushioned in the coil with a vacuum beanbag to prevent motion.

The fMRI pre-processing steps, conducted with SPM5 (Wellcome Department of Cognitive Neurology, London, UK), included the realignment of inter- and intra-session acquisitions to correct head movement, normalization to a standard template (Montreal Neurological Institute template) to minimize inter-subject morphological variability and convolution with an isotropic Gaussian kernel (FWHM, $8 \mathrm{~mm}$ ) to increase the signal-to-noise ratio. Single subject analysis was performed according to the general linear model. The signal drift across acquisitions was removed with high-pass filter $(128 \mathrm{~Hz})$ and global signal changes by proportional scaling. Statistical parametrical maps of the contrasts of interest were computed for each subject as input values for the group statistics based on random field theory. Corrections for multiple comparisons were used at the group level to determine clusters showing a significant effect in the ANOVA [false discovery rate (FDR)-corrected $P<0.05$ ] with the factors aMT (past, now and future), rMT (relative-past and relative-future) and Self (self-related and non-self-related). Selected clusters were used as regions of interest in order to extract the mean fMRI signal change for each condition in every subject.

\section{Results}

\section{Experiment 1: mental time with respect to face images}

\section{Behaviour}

Data showed that RTs and ERs in Past and Future self-locations were significantly higher than in the Now self-location (aMT effect: $F_{2,22}=4.52, P<0.03 ; F_{2,22}=12.4, P<0.001$, respectively; Fig. $2 \mathrm{~A}$ and $\mathrm{B}$ ). In addition, these behavioural data also showed another effect, i.e. participants were always faster for relative-future than for relativepast faces, across all self-locations (rMT effect: RTs, $F_{1,11}=32.1$, $P<0.001$; ERs, $F_{1,11}=12.4, P<0.01$; Fig. $2 \mathrm{~A}$ and B). These effects are independent of one another and we found no interactions among them $\left(\mathrm{aMT} \times \mathrm{rMT}\right.$ : RTs, $F_{2,22}=0.15, P>0.8$; ERs, $F_{2,22}=1.69$, $P>0.2$ ). Behavioural results also showed an effect of Self (selfrelated lower than non-self-related; RTs, $F_{1,11}=5.0, P<0.05$; ERs, $F_{1,11}=16.2, P<0.01$; for more statistics and post-hoc tests, see Supporting information, Table S1). Analysis of the control task also showed an effect of Self, as participants were always faster for self than for non-self faces $\left(t_{1,11}=32.5, P<0.001\right)$. The aMT effect in the present paradigm using faces representing different ages is parallel to previous behavioural data showing an aMT effect with respect to imagination of past and future events (Szpunar et al., 2007; Arzy et al., 2008). Evidence for response facilitation for relative-future faces (rMT effect), leading to faster and more accurate responses, is similar to previously reported findings with respect to past and future events (Arzy et al., 2008). Note that this effect was found independently of the actual age of the presented faces, suggesting that the mental processing of time is future oriented across all investigated self-locations in time (Suddendorf \& Corballis, 1997, 2007; Dudai \& Carruthers, 2005; Schacter et al., 2007; Arzy et al., 2008; Boyer, 2008).

\section{Neuroimaging}

In order to examine the neural basis of MT, brain activity for MT in the Past, Now and Future conditions was compared with brain activity for face discrimination. Analysis for Past, Now and Future selflocations revealed a network of brain regions that were recruited for MT (Table 1). This network included regions in the left inferior frontal cortex [Brodmann area (BA) 9], insular (BA 13) and cingulate (BA 24) cortex bilaterally, right premotor cortex (BA 6) and supplementary motor area, right medial temporal lobe (BA 21), fusiform face area bilaterally (BA 37), right temporo-parietal junction (BA 40), as well as lingual gyrus (BA 19), posterior parietal cortex (BA 7/19) and occipital cortex (BAs 19 and 18) bilaterally.

In a subset of these brain regions, the percent of fMRI signal change showed higher activation for the Past and Future self-locations than for the Now, reflecting the aMT effect in concordance with the behavioural results. These were the inferior frontal and medial temporal cortex $\left[F_{2,22}=4.6, P<0.05 ; F_{2,22}=3.9, P<0.05\right.$, respectively (FDR corrected)] (Fig. 3, supporting Table S2). Significant differences between self-locations in time (aMT effect) were also found in the posterior parietal cortex $\left(F_{2,22}=4.0, P<0.05\right)$. The posterior parietal cortex and insula also reflected response facilitation for relative-future faces, showing lower activation for relative-future than relative-past events (rMT effect: $F_{1,11}=9.6, \quad P<0.02$; $F_{1,11}=7.7, P<0.02$; supporting Table S2) independently of the imagined self-location in time (no interaction of aMT $\times$ rMT: 

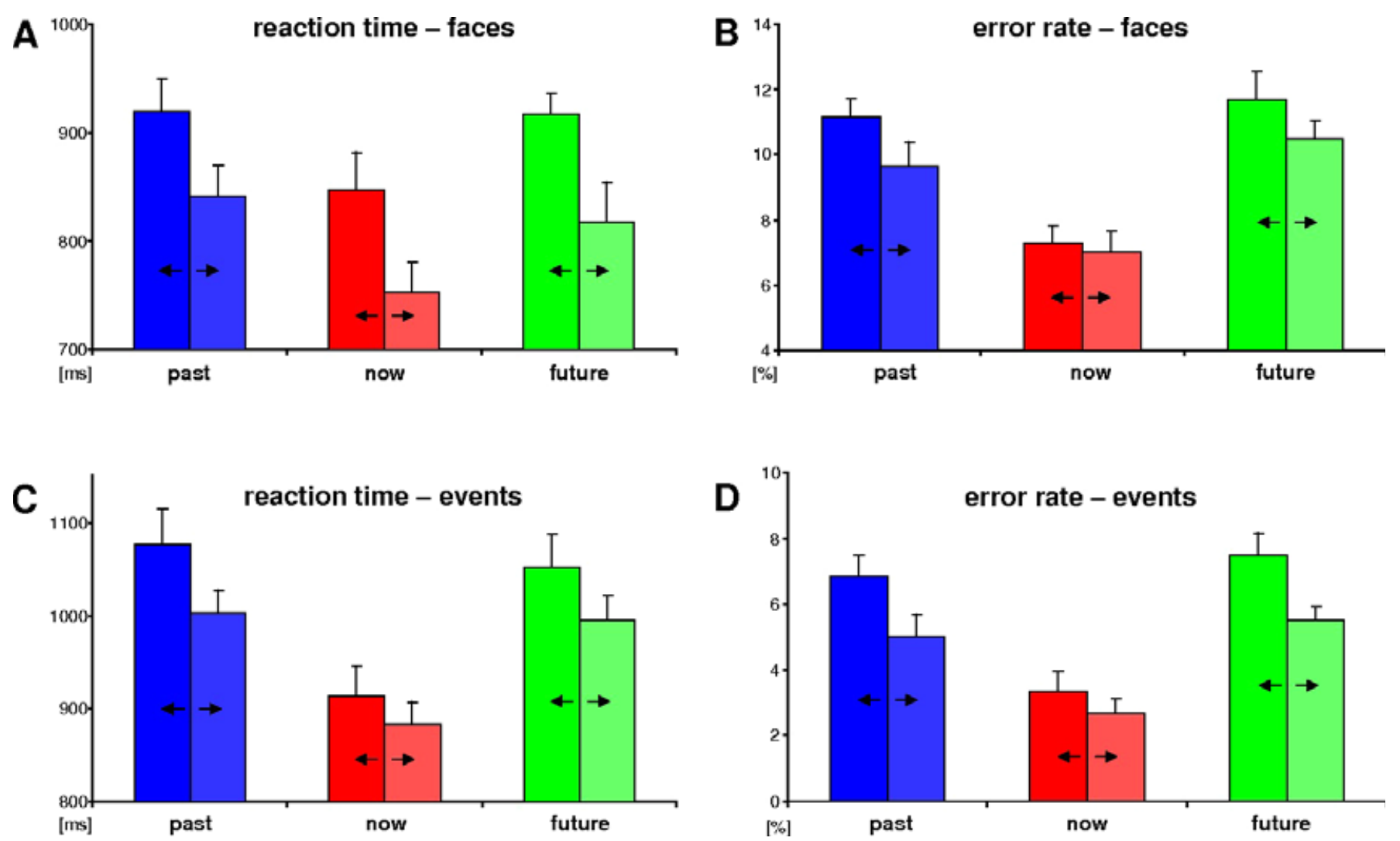

FIG. 2. Behavioural results. RTs and ERs obtained in Experiment 1 (faces, A and B) and Experiment 2 (events, C and D) are plotted separately for Past, Now and Future self-location in time. Error bars indicate intersubject SEM. Both RTs and ERs were found to be significantly higher for the Past and Future with respect to the Now. In addition, in all self-locations in time (Past, Now and Future), RTs and ERs were higher for relative-past (left darker bars) than relative-future (right lighter bars). Note the similarity of these two effects between the two experiments.

TABLE 1. Cerebral network recruited for self-projection in MT

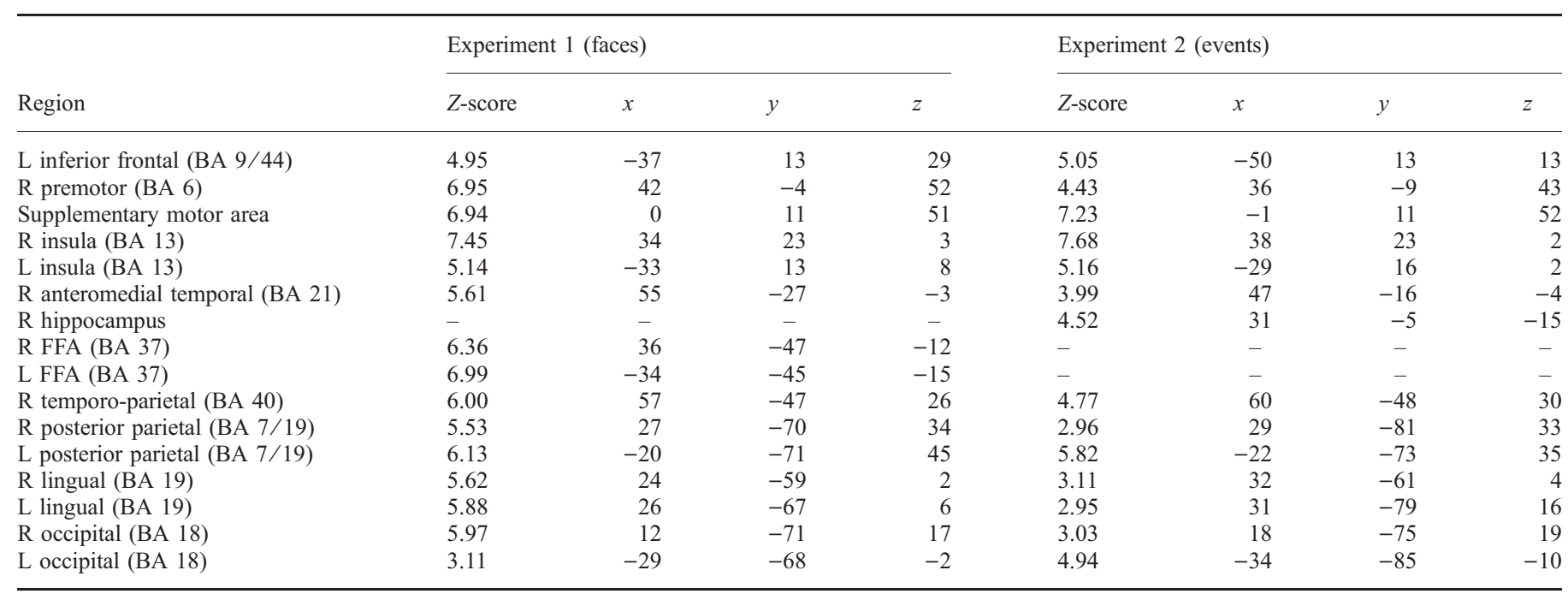

FFA, fusiform face area; L, left; R, right.

$F_{2,22}=0.29, P>0.7 ; F_{2,22}=0.11, P>0.8 ; F_{2,22}=0.07, P>0.9$, respectively; Fig. 3, supporting Table S2). fMRI signal change analysis at the right temporo-parietal junction showed enhanced activity when imagined stimuli were temporally close to the present moment (temporal distance effect; significant interaction aMT $\times$ rMT: $\left.F_{2,22}=6.61, P<0.01\right)$. We thus found a higher activation for relative- future than relative-past in the Past condition, similar activation for relative-past and relative-future in the Now condition and higher activation for relative-past than relative-future in the Future condition with no effects of aMT $\left(F_{2,22}=0.05, P>0.94\right)$ or $\operatorname{rMT}\left(F_{1,11}=0.04\right.$, $P>0.95)$. Finally, comparison of self-related and non-self-related stimuli revealed brain activations at the medial prefrontal (BA 9), 


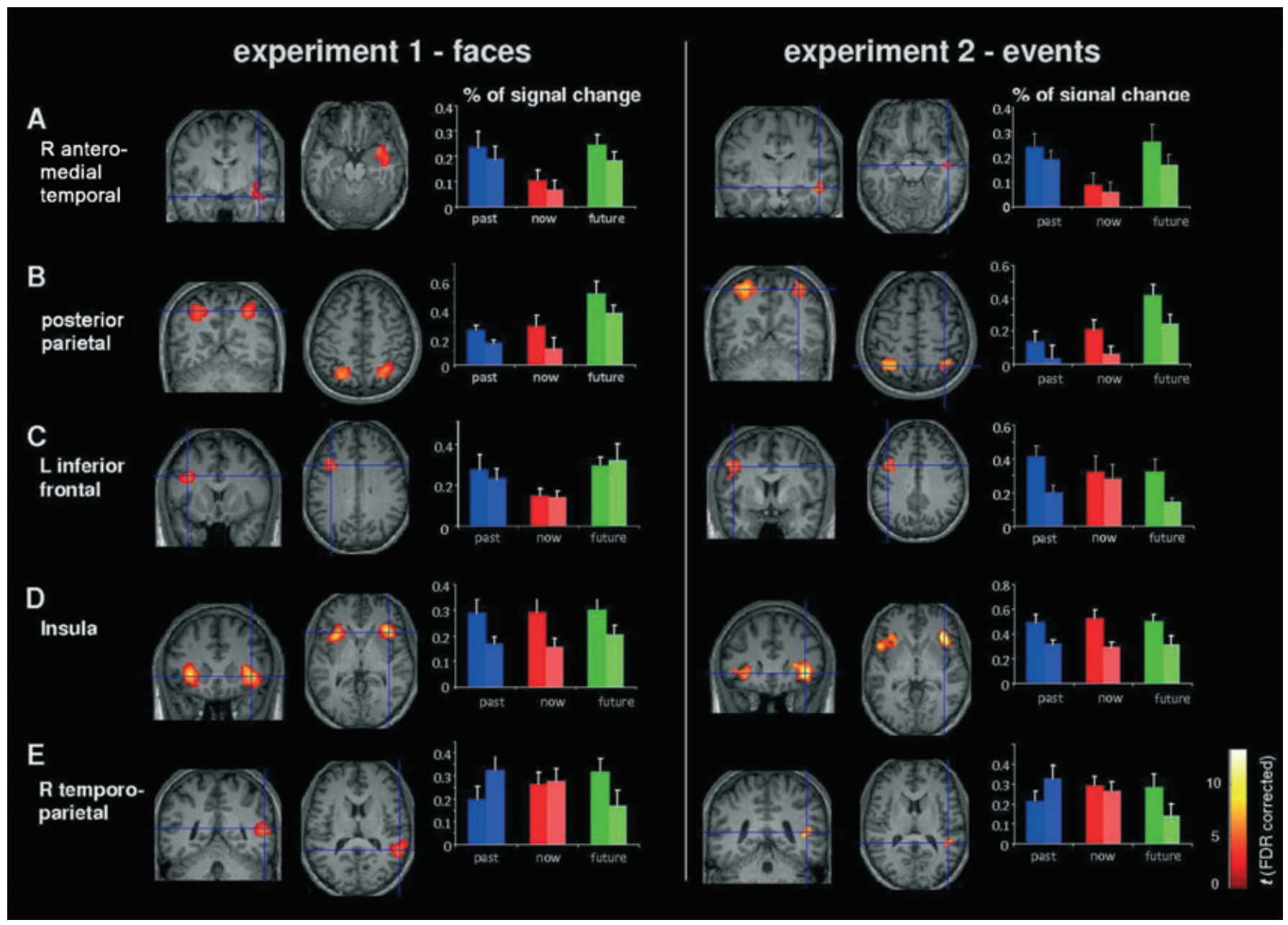

FIG. 3. Neuroimaging results. (A-E) Brain regions commonly engaged in the Past, Now and Future self-locations in time relative to the control task in Experiment 1 (faces, left columns) and Experiment 2 (events, right columns). For all regions significant in these analyses, past, now and future were engaged more than the control task at a threshold of $P<0.05$ (FDR corrected). Percent signal change data associated with each of these conditions are plotted. Note the similarity in brain regions and signal change between the two experiments (see Table 1 for coordinates and Brodmann areas, as well as for regions that did not show a signal difference between conditions).

temporo-parietal (BA 40) and posterior parietal (BA 19/7) cortex bilaterally (self effect; all p's $<0.005$; supporting Fig. S1), similar to previous results (e.g. Ruby \& Decety, 2001; Johnson et al., 2002; Vogeley \& Fink, 2003; David et al., 2006). Percent of fMRI signal change analysis for the other implicated brain regions did not show any of these or other differences.

\section{Experiment 2: events}

\section{Behaviour}

Data showed that RTs and ERs in Past and Future self-locations were significantly higher than in the Now (aMT effect: $F_{2,22}=13.0$, $P<0.001 ; F_{2,22}=23.5, P<0.01$, respectively; Fig. $2 \mathrm{C}$ and $\left.\mathrm{D}\right)$ (Szpunar et al., 2007; Arzy et al., 2008). Replicating previous findings (Arzy et al., 2008), there was also a behavioural rMT effect; participants were faster for relative-future than for relative-past events, with respect to their imagined temporal self-location, across all selflocations (RTs: $F_{1,11}=6.5, P<0.05$; ERs: $F_{1,11}=11.68, P<0.01$; Fig. $2 \mathrm{C}$ and $\mathrm{D}$ ), with no interaction $\left(\mathrm{aMT} \times \mathrm{rMT}\right.$ : RTs, $F_{2,22}=2.6$, $P>0.1$; ERs, $\left.F_{2,22}=2.54, P>0.1\right)$ Behavioural results also showed an effect of Self (self-related lower than non-self-related; RTs:
$F_{1,11}=6.4, P<0.05$; ERs: $F_{1,11}=12.4, P<0.01$; for more statistical analyses and post-hoc tests, see supporting Table S3), replicating previous findings (Arzy et al., 2008). Analysis of the control task also showed a significant difference between self and non-self events $\left(t_{1,11}=77.7, P<0.001 ;\right.$ Self effect $)$. These effects replicate those reported in Experiment 1 with respect to faces across all investigated self-locations in time.

\section{Neuroimaging}

Analysis identical to the analysis performed in Experiment 1 was conducted to examine aMT, rMT, Self and temporal distance effects with respect to events. Brain activity for MT in the Past, Now and Future conditions was compared with brain activity for event discrimination. This analysis revealed engagement of a network of brain regions similar to those found in Experiment 1 (Table 1, Fig. 3, right columns), including the left inferior frontal cortex (BA 9), insular (BA 13) cortex bilaterally, right premotor cortex (BA 6), supplementary motor area, right anteromedial temporal lobe (BA 21) and right hippocampus, right temporo-parietal junction (BA 40) and posterior parietal cortex (BA 7/19), lingual (BA 19) and occipital cortex (BAs 19 and 18) bilaterally. 
experiment 1 - faces
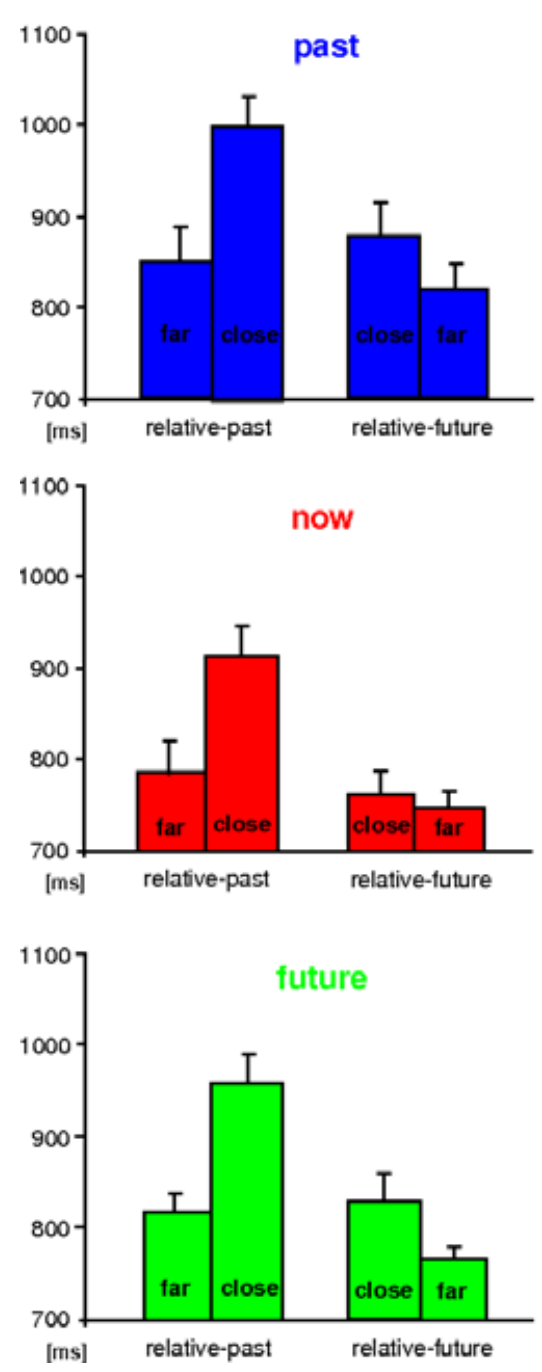

experiment 2 - events
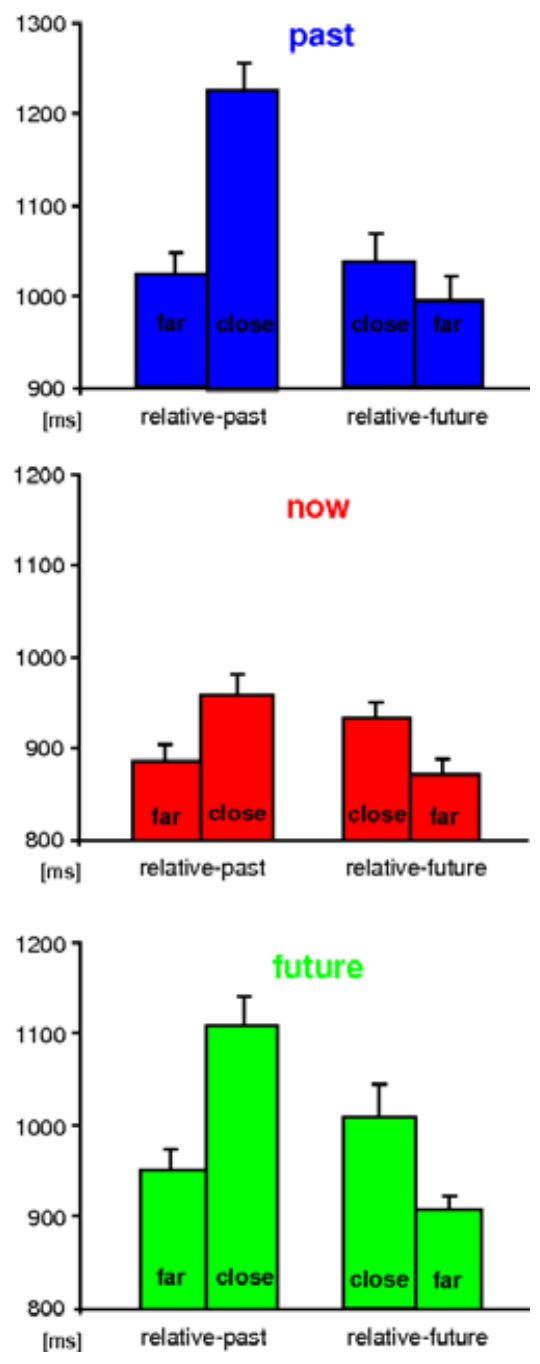

FIG. 4. Temporal distance effect. RTs obtained in Experiment 1 (faces, left column) and Experiment 2 (events, right column) are plotted separately for Past, Now and Future self-location in time, and are grouped according to their temporal distance from the imagined self-location in time (4 and 12 years in Experiment 1 ; $1-10$ and 11-20 years in Experiment 2). Error bars indicate intersubject SEM. RTs were found to be significantly higher for shorter temporal distances from the imagined self-location in time. Note the similarity of these two effects between the two experiments.

The percent of fMRI signal change in the posterior parietal cortex bilaterally and the right anteromedial temporal lobe showed a significant aMT effect (differences between self-locations in time), similar to findings in Experiment 1 [aMT effect: $F_{2,22}=5.98, P<0.01$; $F_{2,22}=4.10, P<0.05$, respectively (FDR corrected); Fig. 3, right columns; supporting Table S4]. In agreement with Experiment 1, the percent of signal change in the posterior parietal cortex and insular cortex bilaterally, as well as the left inferior frontal cortex, showed significantly lower activation for relative-future than relative-past events (rMT effect: $F_{1,11}=9.60, P<0.01 ; F_{1,11}=11.27, P<0.01$; $F_{1,11}=13.48, P<0.01$; Fig. 3 , right columns) independently of the imagined self-location in time (no aMT $\times$ rMT interaction, supporting Table S4). As in Experiment 1, signal change analysis at the right temporo-parietal junction showed higher activation for relative-future than relative-past in the Past condition, similar activation for relativepast and relative-future in the Now condition, and higher activation for relative-past than relative-future in the Future condition (temporal distance effect; interaction aMT $\times$ rMT: $F_{2,22}=5.76, P<0.01$; supporting Table S4). Finally, similarly to Experiment 1, comparison of self-related and non-self-related stimuli revealed brain activations at the medial prefrontal (BA 9) and temporo-parietal (BA 40) cortex bilaterally with left predominance, and at the left posterior parietal cortex (BA 19/7) (Self effect; all p's < 0.005; supporting Fig. S1), similar to previous studies (e.g. Ruby \& Decety, 2001; Johnson et al., 2002; Vogeley \& Fink, 2003; David et al., 2006). The percent of fMRI signal change analysis for the other implicated brain regions did not show any such differences. Differences in brain activation between the two experiments were found at the fusiform face area (activated for faces but not events) and right hippocampus (activated for events but not faces), with no effects of aMT, rMT, Self or temporal distance. This is probably related to the implication of the fusiform face area in face recognition (Kanwisher et al., 1997) and the right hippocampus in event recall (Okuda et al., 2003; Squire et al., 2004; Addis et al., 2007).

\section{Discussion}

We studied MT by asking participants to project themselves to past, now or future self-locations (aMT) and to indicate whether the 
presented faces or events refer to time-points before (relative-past) or after (relative-future) the imagined self-location in time (rMT). These two aspects of MT were reflected at the behavioural level, as RTs and ERs for both faces and events were higher for past and future selflocations in time than for the present time (aMT effect). In addition, in all self-locations in time, participants were faster and more accurate for relative-future than relative-past (rMT effect). This was found for faces as well as for events. These effects were also borne out at the neural level, activating a network common for both experiments consisting of the right anteromedial temporal lobe, posterior parietal cortex bilaterally, left inferior frontal cortex, insula bilaterally and right temporo-parietal junction. Although the two types of stimuli (faces and events) are traditionally employed to test two different perceptual and cognitive faculties and recruit different brain mechanisms in the fusiform face area (Experiment 1) (Kanwisher et al., 1997) and in the medial-temporal lobe (Experiment 2) (Squire et al., 2004), the present data show that common brain mechanisms related to self-projection in time were employed in responses to both stimuli.

\section{Memory and absolute mental time}

Seminal work regarded MT as 'episodic thinking', a combination of episodic memory recall and episodic future imagination (Tulving, 1985, 2002; Atance \& O'Neill, 2001; Levine, 2004; Levine et al., 2004; Schacter et al., 2007; Suddendorf \& Corballis, 2007), whereas more recent authors proposed that MT can be seen as 'self-projection' in time, similarly to 'self-projection' in space, emphasizing the role of visuo-spatial perspective taking, spatial navigation and mental imagery in MT (Buckner \& Carroll, 2007; Hassabis et al., 2007; Arzy et al., 2008). Regarding the first approach, episodic thinking relies extensively on brain mechanisms in the anteromedial temporal lobe, which is a crucial structure for processing both semantic and episodic information, as found in clinical and neuroimaging studies (Corkin, 2002; Levine, 2004; Squire et al., 2004; Svoboda et al., 2006). In the present study, activity in the anteromedial temporal lobe reflected aMT not only in Experiment 2, employing episodic memory, but also in Experiment 1, employing memory processes related to faces. This points to the role of the anteromedial temporal lobe in MT not only with respect to memorizing and imagining events but also with respect to memorizing and imagining one's faces from different points of time. With respect to aspects concerning 'self-projection' in MT (Buckner \& Carroll, 2007), the anteromedial temporal lobe was found to be a key region in both egocentric and allocentric spatial representation for both future imagery and past retrieval (Hassabis et al., 2007; Burgess, 2008; Spreng et al., 2009). Our findings suggest the implication of this region in aspects of MT that are related to self-projection in time.

Analysis of the event-related fMRI signal also showed an aMT effect in the left inferior frontal gyrus. The left inferior frontal cortex was shown to be involved in several different memory functions, including working memory, semantic memory and episodic memory (Dolan \& Fletcher, 1997; Nyberg et al., 2003), as well as the encoding, sorting and retrieving of memory items (Dolan \& Fletcher, 1997; Badre \& Wagner, 2007). Mesulam (2002) highlighted the role of this region in transposing one's 'reference point (or self-location) from self to other, from here to there, and from now to then'. Tulving (1985) specified that the frontal cortex is important for the awareness of one's existence across subjective time and Fuster (1989) proposed it to be essential for re-experiencing the past and foreseeing the future. Others emphasized the role of the inferior frontal cortex in processing self-referential information (Gusnard et al., 2001; Maguire, 2001; Johnson et al., 2002; Okuda et al., 2003; Gilboa, 2004; Gilboa et al.,
2004; Addis et al., 2007; Szpunar et al., 2007), including autobiographical memory and personal future events (although the activations in some of these studies were more medial and dorsal than those reported here).

Differences between self-locations in time were also found in the posterior parietal cortex in both experiments. Similarly to the anteromedial temporal lobe, the posterior parietal cortex is also implicated in both episodic thinking and spatial representation (Addis et al., 2007; Buckner \& Carroll, 2007; Szpunar et al., 2007; Arzy et al., 2008; Spreng et al., 2009). With respect to episodic thinking, the posterior parietal cortex was implicated in visual imagery of the retrieved or imagined episodes (Gilboa, 2004; Gilboa et al., 2004; Levine, 2004; Levine et al., 2004; Svoboda et al., 2006; Addis et al., 2007; Szpunar et al., 2007). In addition, neuroimaging studies reported activation of the posterior parietal cortex in mental imagery in self- and space-related tasks, including motor imagery, mental rotation and mental navigation as well as the distinction between egocentric and allocentric reference frames (Zacks et al., 1999; Ruby \& Decety, 2001; Vogeley \& Fink, 2003; Arzy et al., 2006; David et al., 2006; Zacks, 2008). These previous data and the activation of this region in both experiments emphasize the role of spatial aspects in MT-related brain processes that we tested here using self-projection in MT, requiring episodic thinking, mental-imagery and self. Taken together, aMT for faces and events recruits brain mechanisms in the anteromedial temporal lobe, posterior parietal cortex and inferior frontal cortex related to episodic thinking, mental imagery, spatial representation and the self jointly contributing to self-projection in time.

\section{Future anticipation and relative mental time}

Participants were always faster and more accurate for relative-future than relative-past (rMT effect), independent of their temporal selflocation and independent of the employed stimulus (faces or events). This provides experimental evidence for proposals that MT is used principally to anticipate future occurrences (Ingvar, 1985; Suddendorf \& Corballis, 1997, 2007; Dudai \& Carruthers, 2005; Boyer, 2008). Evolutionary considerations suggest that vivid episodic thinking may be crucial for human decision making (Suddendorf \& Corballis, 1997, 2007; Boyer, 2008), enabling faster judgements related to future than to past. The behavioural rMT effect was also expressed at the neural level by the lower activations that we found for relative-future as compared with relative-past found in the posterior parietal cortex and insula. These structures have been proposed to combine aspects of MT related to visual imagery and emotions. Implication of visual imagery in episodic future thinking and past recall may rely on brain mechanisms in the posterior parietal cortex, in agreement with previous neuroimaging studies (Maguire, 2001; Conway et al., 2002; Svoboda et al., 2006; Addis et al., 2007; Szpunar et al., 2007; Spreng et al., 2009). Emotional engagement with past or future events is an important aspect of MT (Sharot et al., 2004; Boyer, 2008). We argue that emotional aspects of MT might be reflected in the activation of the insular cortex and might also reflect emotional aspects related to the self that have been called 'self-referential emotions' (Zinck, 2008). The rMT effect in insular activation might reflect misalignment between one's past emotional affective state and one's future imagination (Sharot et al., 2004; Boyer, 2008), as well as higher emotional load with respect to past faces and events. The insula was proposed to provide the basis for the subjective image of the self as a sentient entity and to accumulate updated information about one's body (Craig, 2002, 2003, 2004). The insular cortex has major 
connections to the anterior cingulate cortex, amygdala, hypothalamus and prefrontal and orbito-frontal cortices (Augustine, 1996; Craig, 2003, 2004; Nagai et al., 2007). Functional neuroimaging studies reveal this cortex to be activated by information regarding the body or self, in order to regulate the body states accordingly (Craig, 2003, 2004). This is compatible with the present results showing insular activation for self-projection in time and the rMT effect, proposing insular implication in future regulation of body states.

\section{Temporal distance in mental time}

Activation at the right temporo-parietal junction showed a symmetrical distribution of the fMRI signal change as a function of the temporal distance of the faces/events from the present time. Thus, activation was increased for relative-future as compared with relative-past in the Past condition, activities were similar to relative-past and relativefuture in the Now, and higher activity was found for relative-past as compared with relative-future in the Future condition (Fig. 3E). A similar temporal distance effect of symmetrical distribution was found in previous behavioural studies (D'Argembeau \& Van der Linden, 2004; Spreng \& Levine, 2006; Arzy et al., 2009) and in our behavioural data when we plotted the percentage of correct recall and RTs as a function of the temporal distance between the imagined selflocation and the past and future life events or faces (Fig. 4). In all conditions, RTs and ERs were aligned with the temporal distance of the presented face/event from the imagined self-location in time. This may be compatible with the role of the temporo-parietal junction in coordinating the relation between one's self-location in space and different external reference points (Ruby \& Decety, 2001; Vogeley \& Fink, 2003). In particular, previous studies have shown that the temporo-parietal junction is a key structure for maintaining the relations between one's actual self-location in space ('here') and an imagined one ('there') (Zacks et al., 1999; Ruby \& Decety, 2001; Vogeley \& Fink, 2003; Blanke et al., 2005; Arzy et al., 2006; David et al., 2006; Corradi-Dell'acqua et al., 2008) and that temporoparietal activation may depend on the spatial angle (or rotational distance) between the subject's actual and imagined self-location (Blanke et al., 2005; Arzy et al., 2006). Based on these previous and present behavioural and neural data, we speculate that this may be similar, at least in part, when imaging temporal relationships between habitual self-location in time (Now) and imagined ones (Past and Future) that were investigated in the present study and that the temporo-parietal cortex is a key structure for encoding the self in time and space.

In conclusion, the present two experiments show MT to include three independent components. (i) Similarity between self-projection in time to past and future is encoded in the anteromedial temporal lobe and inferior frontal cortex. (ii) Relative facilitation of future judgements with respect to one's past is encoded in the posterior parietal cortex and insula. (iii) The dependency of MT on the temporal distance between one's self-location in time and memorized/imagined faces and events is encoded at the temporo-pariatal junction. The recruited brain regions were highly similar in both experiments, for faces and events, suggesting that the distributed brain mechanisms of MT as tested here are independent of whether actual life episodes have to be re-experienced/pre-experienced. The multifunctional character of this distributed network shows the complexity of MT, incorporating classical memory structures and neural mechanisms related to mental imagery and the self, allowing the description of MT as a multifaceted cognitive faculty, essential to anticipate and pilot future behaviour according to the present perception of past experiences.

\section{Supporting Information}

Additional supporting information may be found in the online version of this article:

Fig. S1. Temporal distance effect. RTs obtained in Experiment 1 and Experiment 2 are plotted separately for Past, Now and Future selflocation in time, and are grouped according to their temporal distance from the imagined self-location in time.

Table S1. Statistical results and post-hoc tests for RTs in experiment 1 (faces).

Table S2. Statistical results and post-hoc tests for neuroimaging results in experiment 1.

Table S3. Statistical results and post-hoc tests for RTs in experiment 2 (events).

Table S4. Statistical results and post-hoc tests for neuroimaging results of experiment 2 .

Please note: As a service to our authors and readers, this journal provides supporting information supplied by the authors. Such materials are peer-reviewed and may be re-organized for online delivery, but are not copy-edited or typeset by Wiley-Blackwell. Technical support issues arising from supporting information (other than missing files) should be addressed to the authors.

\section{Acknowledgements}

We thank Professor Salvatore M. Aglioti (University of Rome 'La Sapienza', Italy) for helpful comments on a previous version of the manuscript and Dr Stéphane Simon (The Center for Biomedical Imaging of Geneva and Lausanne, Switzerland) for his assistance in data analysis.

\section{Abbreviations}

aMT, absolute mental time; ER, error rate; FDR, false discovery rate; fMRI, functional magnetic resonance imaging; MT, mental time; rMT, relative mental time; RT, reaction time.

\section{References}

Addis, D.R., Wong, A.T. \& Schacter, D.L. (2007) Remembering the past and imagining the future: common and distinct neural substrates during event construction and elaboration. Neuropsychologia, 45, 1363-1377.

Arzy, S., Thut, G., Mohr, C., Michel, C.M. \& Blanke, O. (2006) Neural basis of embodiment: distinct contributions of temporoparietal junction and extrastriate body area. J. Neurosci., 26, 8074-8081.

Arzy, S., Molnar-Szakacs, I. \& Blanke, O. (2008) Self in time: imagined selflocation influences neural activity related to mental time travel. J. Neurosci., 28, 6502-6507.

Arzy, S., Adi-Japha, E. \& Blanke, O. (2009) The mental time line: an analogue of the mental number line in the mapping of life events. Conscious. Cogn., doi:10.1016/j.concog.2009.05.007.

Atance, C.M. \& O’Neill, D.K. (2001) Episodic future thinking. Trends Cogn. Sci., 5, 533-539.

Augustine, J.R. (1996) Circuitry and functional aspects of the insular lobe in primates including humans. Brain Res. Brain Res. Rev., 22, 229-244.

Badre, D. \& Wagner, A.D. (2007) Left ventrolateral prefrontal cortex and the cognitive control of memory. Neuropsychologia, 45, 2883-2901.

Blanke, O., Mohr, C., Michel, C.M., Pascual-Leone, A., Brugger, P., Seeck, M., Landis, T. \& Thut, G. (2005) Linking out-of-body experience and self processing to mental own-body imagery at the temporoparietal junction. J. Neurosci., 25, 550-557.

Boyer, P. (2008) Evolutionary economics of mental time travel? Trends Cogn. Sci., 12, 219-224.

Buckner, R.L. \& Carroll, D.C. (2007) Self-projection and the brain. Trends Cogn. Sci., 11, 49-57.

Burgess, N. (2008) Spatial cognition and the brain. Ann. NY Acad. Sci., 1124, 77-97.

Conway, M.A., Pleydell-Pearce, C.W., Whitecross, S. \& Sharpe, H. (2002) Brain imaging autobiographical memory. Psychol. Learn. Motiv., 41, 229264. 
Corkin, S. (2002) What's new with the amnesic patient H.M.? Nat. Rev., 3, 153-160.

Corradi-Dell'acqua, C., Ueno, K., Ogawa, A., Cheng, K., Rumiati, R.I. \& Iriki, A. (2008) Effects of shifting perspective of the self: an fMRI study. Neuroimage, 40, 1902-1911.

Craig, A.D. (2002) How do you feel? Interoception: the sense of the physiological condition of the body. Nat. Rev., 3, 655-666.

Craig, A.D. (2003) Interoception: the sense of the physiological condition of the body. Curr. Opin. Neurobiol., 13, 500-505.

Craig, A.D. (2004) Human feelings: why are some more aware than others? Trends Cogn. Sci., 8, 239-241.

D'Argembeau, A. \& Van der Linden, M. (2004) Phenomenal characteristics associated with projecting oneself back into the past and forward into the future: influence of valence and temporal distance. Conscious. Cogn., 13, 844-858.

David, N., Bewernick, B.H., Cohen, M.X., Newen, A., Lux, S., Fink, G.R., Shah, N.J. \& Vogeley, K. (2006) Neural representations of self versus other: visual-spatial perspective taking and agency in a virtual ball-tossing game. Proc. Natl Acad. Sci. USA, 18, 898-910.

Dolan, R.J. \& Fletcher, P.C. (1997) Dissociating prefrontal and hippocampal function in episodic memory encoding. Nature, 388, 582-585.

Dudai, Y. \& Carruthers, M. (2005) The Janus face of Mnemosyne. Nature, 434, 567.

Fellows, L.K. \& Farah, M.J. (2005) Dissociable elements of human foresight: a role for the ventromedial frontal lobes in framing the future, but not in discounting future rewards. Neuropsychologia, 43, 1214-1221.

Fuster, J.M. (1989) The Prefrontal Cortex: Anatomy, Physiology, and Neuropsychology of the Frontal Lobe. Raven Press, Philadelphia, New York.

Gilbert, D.T. \& Wilson, T.D. (2007) Prospection: experiencing the future. Science, 317, 1351-1354.

Gilboa, A. (2004) Autobiographical and episodic memory - one and the same? Evidence from prefrontal activation in neuroimaging studies. Neuropsychologia, 42, 1336-1349.

Gilboa, A., Winocur, G., Grady, C.L., Hevenor, S.J. \& Moscovitch, M. (2004) Remembering our past: functional neuroanatomy of recollection of recent and very remote personal events. Cereb. Cortex, 14, 1214-1225.

Gusnard, D.A., Akbudak, E., Shulman, G.L. \& Raichle, M.E. (2001) Medial prefrontal cortex and self-referential mental activity: relation to a default mode of brain function. Proc. Natl Acad. Sci. USA, 98, 4259-4264.

Hassabis, D., Kumaran, D., Vann, S.D. \& Maguire, E.A. (2007) Patients with hippocampal amnesia cannot imagine new experiences. Proc. Natl Acad. Sci. USA, 104, 1726-1731.

Ingvar, D.H. (1985) "Memory of the future": an essay on the temporal organization of conscious awareness. Hum. Neurobiol., 4, 127-136.

Johnson, S.C., Baxter, L.C., Wilder, L.S., Pipe, J.G., Heiserman, J.E. \& Prigatano, G.P. (2002) Neural correlates of self-reflection. Brain, 125, 18081814

Kanwisher, N., McDermott, J. \& Chun, M.M. (1997) The fusiform face area: a module in human extrastriate cortex specialized for face perception. J. Neurosci., 17, 4302-4311.

Levine, B. (2004) Autobiographical memory and the self in time: brain lesion effects, functional neuroanatomy, and lifespan development. Brain Cogn., 55, 54-68.
Levine, B., Turner, G.R., Tisserand, D., Hevenor, S.J., Graham, S.J. \& McIntosh, A.R. (2004) The functional neuroanatomy of episodic and semantic autobiographical remembering: a prospective functional MRI study. J. Cogn. Neurosci., 16, 1633-1646.

Maguire, E.A. (2001) Neuroimaging studies of autobiographical event memory Philos. Trans. R. Soc. Lond. B Biol. Sci., 356, 1441-1451.

Mesulam, M.M. (2002) The human frontal lobes: transcending the default mode through contingent encoding. In Stuss, D.T. \& Knight, R.T. (Eds), Principles of Frontal Lobe Function. Oxford University Press, Oxford, pp. 8-30.

Nagai, M., Kishi, K. \& Kato, S. (2007) Insular cortex and neuropsychiatric disorders: a review of recent literature. Eur. Psychiatry, 22, 387-394.

Nyberg, L., Marklund, P., Persson, J., Cabeza, R., Forkstam, C., Petersson, K.M. \& Ingvar, M. (2003) Common prefrontal activations during working memory, episodic memory, and semantic memory. Neuropsychologia, 41, 371-377.

Okuda, J., Fujii, T., Ohtake, H., Tsukiura, T., Tanji, K., Suzuki, K., Kawashima, R., Fukuda, H., Itoh, M. \& Yamadori, A. (2003) Thinking of the future and past: the roles of the frontal pole and the medial temporal lobes. Neuroimage, 19, 1369-1380.

Ruby, P. \& Decety, J. (2001) Effect of subjective perspective taking during simulation of action: a PET investigation of agency. Nat. Neurosci., 4, 546-550.

Schacter, D.L., Addis, D.R. \& Buckner, R.L. (2007) Remembering the past to imagine the future: the prospective brain. Nat. Rev., 8, 657-661.

Sharot, T., Delgado, M.R. \& Phelps, E.A. (2004) How emotion enhances the feeling of remembering. Nat. Neurosci., 7, 1376-1380.

Spreng, R.N. \& Levine, B. (2006) The temporal distribution of past and future autobiographical events across the lifespan. Mem. Cognit., 34, 1644-1651.

Spreng, R.N., Mar, R.A. \& Kim, A.S. (2009) The Common Neural Basis of Autobiographical Memory, Prospection, Navigation, Theory of Mind and the Default Mode: a Quantitative Meta-analysis. J. Cogn. Neurosci., 21, 489-510.

Squire, L.R., Stark, C.E. \& Clark, R.E. (2004) The medial temporal lobe. Annu. Rev. Neurosci., 27, 279-306.

Suddendorf, T. \& Corballis, M.C. (1997) Mental time travel and the evolution of the human mind. Genet. Soc. Gen. Psychol. Monogr., 123, 133-167.

Suddendorf, T. \& Corballis, M.C. (2007) The evolution of foresight: what is mental time travel, and is it unique to humans? Behav. Brain Sci., 30, 299313; discussion 313-251.

Svoboda, E., McKinnon, M.C. \& Levine, B. (2006) The functional neuroanatomy of autobiographical memory: a meta-analysis. Neuropsychologia, 44, 2189-2208.

Szpunar, K.K., Watson, J.M. \& McDermott, K.B. (2007) Neural substrates of envisioning the future. Proc. Natl Acad. Sci. USA, 104, 642-647.

Tulving, E. (1985) Memory and consciousness. Can. Psychol., 26, 1-12.

Tulving, E. (2002) Episodic memory: from mind to brain. Annu. Rev. Psychol., 53, $1-25$.

Vogeley, K. \& Fink, G.R. (2003) Neural correlates of the first-personperspective. Trends Cogn. Sci., 7, 38-42.

Zacks, J.M. (2008) Neuroimaging studies of mental rotation: a meta-analysis and review. J. Cogn. Neurosci., 20, 1-19.

Zacks, J., Rypma, B., Gabrieli, J.D., Tversky, B. \& Glover, G.H. (1999) Imagined transformations of bodies: an fMRI investigation. Neuropsychologia, 37, 1029-1040.

Zinck, A. (2008) Self-referential emotions. Conscious. Cogn., 17, 496-505. 